九州大学学術情報リポジトリ

Kyushu University Institutional Repository

\title{
Triage with RFID Tags for Massive Incidents
}

Inoue, Sozo

Kyushu University Library

Sonoda, Akihito

Graduate School of Information Science and Electrical Engineering, Kyushu University

Yasuura, Hiroto

Faculty of Information Science and Electrical Engineering, Kyushu University

http://hdl. handle. net/2324/10051

出版情報 : RFID Handbook: Applications, Technology, Security, and Privacy, pp.329-349，2008-0317. CRC Press

バージョン :

権利関係 : 


\title{
Triage with RFID Tags for Massive Incidents
}

\author{
Sozo INOUE \\ Library, Kyushu University, \\ Akihito SONODA \\ Graduate School of Information Science and Electrical Engineering, Kyushu University \\ Hiroto YASUURA \\ Faculty of Information Science and Electrical Engineering, Kyushu University
}

\begin{abstract}
In this chapter, a triage system using RFID and its experiment are described. Triage is a procedure used by emergency personnel to ration limited medical resources to massive injured people, in which triage tags, are used to 1: classify and transport the injured effectively, and 2: obtain and publish the state and the scale of the casualty incident. We applied RFID tags and mobile devices with a wireless network to speed up 1 and 2 and to make them accurate, and verified the effectiveness through an experiment assuming as massive as 84 injured people, and demonstrated that it even accelerates the transportation of the injured people.
\end{abstract}

\section{Keywords:}

RFID, triage, mobile device, wireless communication, emergency medical service

\section{Introduction}

In this chapter, a triage system using RFID and mobile devices with a wireless network is proposed, and its advantages are verified through an experiment assuming an incident of massive injured people.

Triage is a procedure used by emergency personnel to ration limited medical resources to massive injured people, in which triage tags, are used to 1 . classify and transport the injured people effectively, and 2. obtain the information about the state and the scale of the casualty incident to publish to the masses, or to utilize for the decision-making such as medical resource procurements. Fig. 1 is a picture of a triage tag.

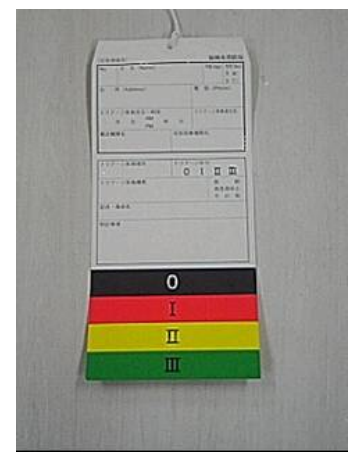




\section{Fig. 1 Triage Tag}

So far, triage is operated manually using paper triage tags, tallies, and radiophones. However, manual operation of 2 becomes failure, inaccuracy, and delay in the information transmission while emergency personnel has priority over treatments for injuries, and causes inefficiency in 1 , which is the essential goal of emergency medical services.

In this chapter, we propose a triage system where RFID tags, which are silicon chips with their IDs, radio frequency functions and some additional logic and memory [1][2], are attached to triage tags. Most of the RFID tags are passive, which means the power is supplied through radio frequency communication from extemal readers. Employed RFID tags in this work are passive and have 1 kilobyte of rewritable memories. Fig. 2 is a picture of an RFID tag we employed.

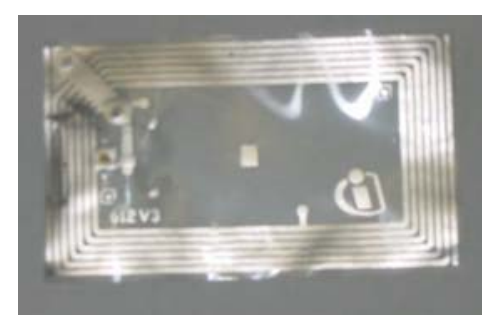

Fig. 2 RFID Tag

Embedding an RFID tag to a triage tag makes the following advantages:

- The terminal the emergency personnel use can identify the injured person by the unique ID value in each RFID tag.

- Rewritable RFID tags provides the storage for the information of the injured person, and the emergency personnel can obtain the information when they are in a place where wireless communication is out of service, such as deep in a mountain or in the underground.

- The time for inputting an injured-person's information can be independent of the network communication, since the information already input so far about the injured person, which is shown to the emergency personnel when they begin to add information, is not obtained through the network, but from the RFID tag.

This application addresses important challenges for pervasive computing: data integrity, that the information should not be lost after being input, input throughput, that the time for inputting injured-person's information should be as short as possible, availability, that emergency personnel should be able to use the system any time, and low latency of communication. These should be as independent of the network status as possible.

In this chapter, we show a realistic solution for the challenges by specializing the network usage in a way that only particular paths are used in particular stages of the workflow by analyzing the workflow and exploiting RFID tags to slim down the possible paths by the following approaches: Input throughput and availability are assured by using RFID tags as local buffer; Data integrity is assured and latency is improved by defining minimum wireless communication areas in the paths in triage workflow.

To evaluate the effectiveness of the system, we performed two experimental performance of triage assuming a complex car crash of 5 cars and about 82 injured people, where one of the performances is done in the current method, and the 
other is using the system. As a result of the experiment, the information collection was accelerated and made more complete by using the system, especially for the information which is utilized in early stages of the triage. The acceleration of the collection also resulted in the acceleration of the transportation of the injured people, which is the most important objective in triage. Moreover, we could estimate the average acceleration of each input to the terminal by employing RFID tag in the experiment was 14.3 seconds.

The rest of the chapter is organized as follows: Section 2 describes triage and challenges for pervasive computing, Section 3 introduces the RFID triage system, Section 4 describes the related work, Section 5 shows the result of a preliminary experiment to complement the experiment, and Section 6 shows the result of the experiment to confirm the effectiveness of the system, and Section 7 concludes the chapter.

\section{Triage, as a Challenge for Pervasive Computing}

We position triage as not only a practical application which requires urgent and continuous improvement since we are facing massive casualty incidents through the news every day, but also a start point for new horizons in pervasive computing concerning unexpected human behavior, rapid deployment, and insufficient computing infrastructure. In this section, we describe the workflow of triage operated by emergency personnel, address technical requirements for triage regarding the paths of the information collected as that of network, and motivate the challenges for pervasive computing hidden in the requirements.

We target on incidents of about 10 to 100 people are injured in a regional place. Examples of such incidents are a crash/derailment/overturn of a train, a crash/fall of a car, a crash/fire of a plane, and terrorism in a subway/building.

\subsection{Triage}

We call the people who are engaged in triage emergency personnel. Fig. 1 shows an abstract workflow of triage.

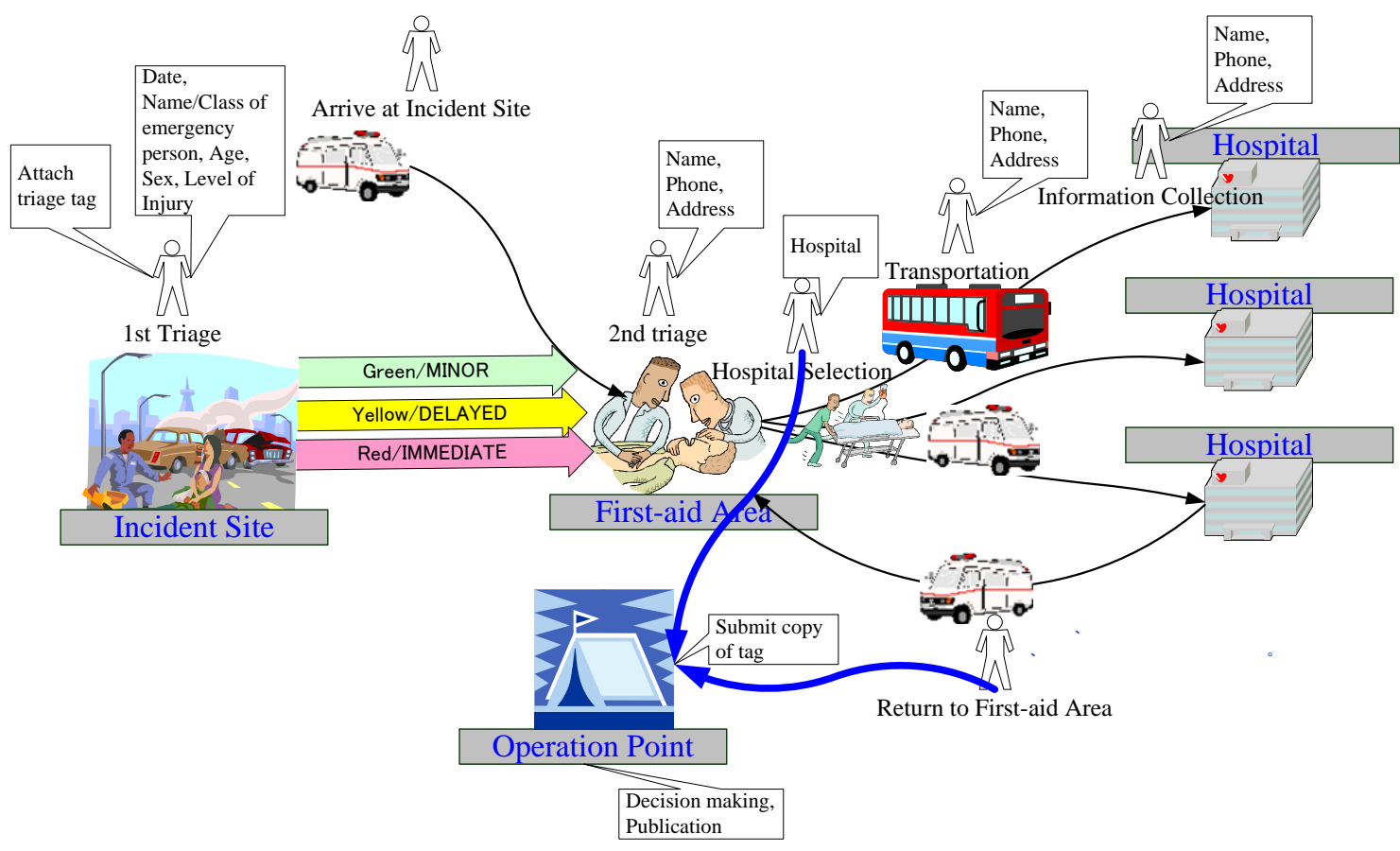

Fig. 3 Workflow in triage 
Current triage is done in the following procedure without using information systems by the emergency personnel.

1. The emergency personnel who arrived to the incident site first establish a first-aid area, which is a safe place for first aid close to the incident site, and an operation point, which is a place to command the triage.

2. (1st triage ) Right after the evacuation of injured people after the incident site is secured by firemen at al., the emergency personnel enter the incident site, and perform $1^{\text {st }}$ triage, in which the personnel distinguish the injury level in about 30 seconds. During the 1st triage, they attach triage tags, each of which has a perforated colored label representing the injury level as the following, to the injured people.

I. Black / DECEASED: is severely injured to die and any hospital care does not help survive.

II. Red / IMMEDIATE: requires immediate surgery or other life-saving intervention, the person is unconscious and has first priority to transport to hospitals.

III. Yellow / DELAYED: requires hospital care and requires to be watched by trained persons, but the person remains conscious and the condition is stable for the moment.

IV. Green / MINOR: may require a doctor's care but not immediately, and the person is able to walk on her/his own.

Simultaneously, the following information is written by the emergency personnel as much as possible:

A) the date of input,

B) the name of the emergency personnel,

C) the category of the emergency personnel, such as doctor, emergency medical technician,

D) rough age of the injured person,

E) the sex of the injured person, and,

F) written version of the injury level.

3. The injured people are moved from the incident site to the first-aid area.

4. (2nd triage) In the first-aid area, besides the injured people treated, $2^{\text {nd }}$ triage are performed if possible, in which the information of the injured people:

G) the name,

H) the phone number,

I) the address,

J) and also the updated information from $1^{\text {st }}$ triage,

are collected as much as the emergency personnel and the injured people can afford to, and written to the triage tags.

5. (Hospital selection) At the exit of the first-aid area, the hospital each injured person is transported is decided and written to the triage tag. A triage tag has carbon copies, and one of them are left to the emergency personnel of the operation point.

6. When an ambulance or a transport vehicle arrives to the first-aid area, the injured people are transported from the first-aid area to the hospital. In the ambulances, the information of the injured people are also collected as much as possible, and written to the triage tags.

7. Other emergency personnel stand by at each hospital, and collect the information of the injured people transported. The ambulance which has once transported several injured people returns to the incident site again, and repeats the transportation of injured people to the hospitals. On returning to the incident site, the ambulance transports the carbon copy of the triage tag at the hospital to the operation point.

8. In the operation point, the information on the carbon copies are collected, and reported to the fire department of the area, which is to be used for decision making, publication, and so on. 


\subsection{Requirements}

The first mission of triage targeted on in the chapter is to transport injured people as quick as possible. As for the mission, information collection of injured people affects to the latency of transportation in 2 ways: troublesome operations for information collection by an emergency personnel causes the latency by disturbing her/his work, and particular types of inputted information should be collected within the early stages since they are used for decisions in the transportation. For the latter, the level of injury and hospital to be transported have to be collected within $1^{\text {st }}$ or $2^{\text {nd }}$ triage prior to the transportation where these information are used, while the other types have not since they are used in the publish afterward. However in the current triage, all of these information must be collected prior to the transportation for the sake of avoiding loss and latency of the collection after the ambulance leaving.

Thus, several technical requirements which can be identified as important challenges for pervasive computing, arise regarding the paths that the information of injured people are collected.

1. (Data integrity) the information of injured people should not be lost after being input. However in current triage, the complete information of injured people is not able to be collected when triage tags fails to be cut off, are lost, or left in the pocket of an emergency person. Moreover, the information filled into a triage tag later than the 1st or 2nd triage stages will be lost when the ambulance never returns to the incident site.

2. (Input throughput) the time for inputting injured-person's information should be as short as possible. Especially, it should be so even if the latency of the network communication becomes large.

3. (Availability) emergency personnel should be able to use the system. Especially, input operations of injured-person's information should be available anytime in the triage, even when the network is unreachable.

4. (Low latency) several types of inputted information should be quickly collected and viewed from the operation point. In current triage, the information filled into a triage tag later than the 1st or 2nd triage stages are not collected until the ambulance which transported the injured person returns to the incident site, even if they are filled in the ambulance or at the hospital, since additional manual communication from the operation point to hospitals or the ambulance increases the work in the operation point.

\subsection{Challenges in pervasive computing}

The requirement listed above are important challenges in pervasive computing which aims one in harmony with real world's circumstance, including unexpected human behavior, rapid deployment, or insufficient computing infrastructure. In this chapter, we motivate these challenges, and address an improvement in a special but realistic case of pervasive computing, besides the improvement of triage by introducing information technology.

Data integrity and latency will be improved by exploiting a wireless network, since the inputted information can be collected through the network, even if emergency personnel does not directly or indirectly give a triage tag to the person who is in charge of collecting the information. However, this stands on the assumption that once inputted information can be reachable to the destination in the network for more than a while. This assumption does not always hold in pervasive computing. Hence, data integrity and low latency in the semi-reachable network is one of the challenges in pervasive computing.

Input throughput and availability against the network are further challenging requirements in pervasive computing. The real world is not of course composed as a set of packets like TCP/IP, but behaves as the real time system. The interaction between the real world and the network in the interface such as mobile devices, sensors, and RFID tags becomes incoherent if the gap is not absorbed in the network nodes in appropriate layers from physical to human workflows. In these sense, independence of network status for real-world interfaces is also one of the attractive challenges in pervasive computing. 
In this chapter, we show a realistic solution for the challenges by specializing the network usage in a way that only particular paths are used in particular stages of the workflow by analyzing the workflow and exploiting RFID tags to slim down the possible paths by the following approaches:

- Input throughput and availability are assured by using RFID tags as local buffer: users can input data by referring the RFID data already input so far and the input device pushes the inputted data to the queue which is sent to the destination independently of the user's operation, as well as writing to the RFID tag.

- Data integrity is assured and latency is improved by providing wireless communication areas at least to the final stages of the paths in triage workflow: hospitals and the operation point. Actually, latency will be more improved if the stages covered with wireless communication areas increases, but we believe it will be better than current triage, as experimented in Section 6.

The requirements in environments of insufficient network infrastructure including disasters are also discussed in mobile and ad hoc networks[14][15][16] with a requirement for quick deployment, but our approach of using RFID tags for input throughput and availability is unique, while most of the RFID applications in the literature use RFID tags as a device to identify objects or people by embedding unique IDs.

\section{RFID Triage System}

In this section, we describe the triage system using RFID Tags. REF_Ref165807902 ¥h

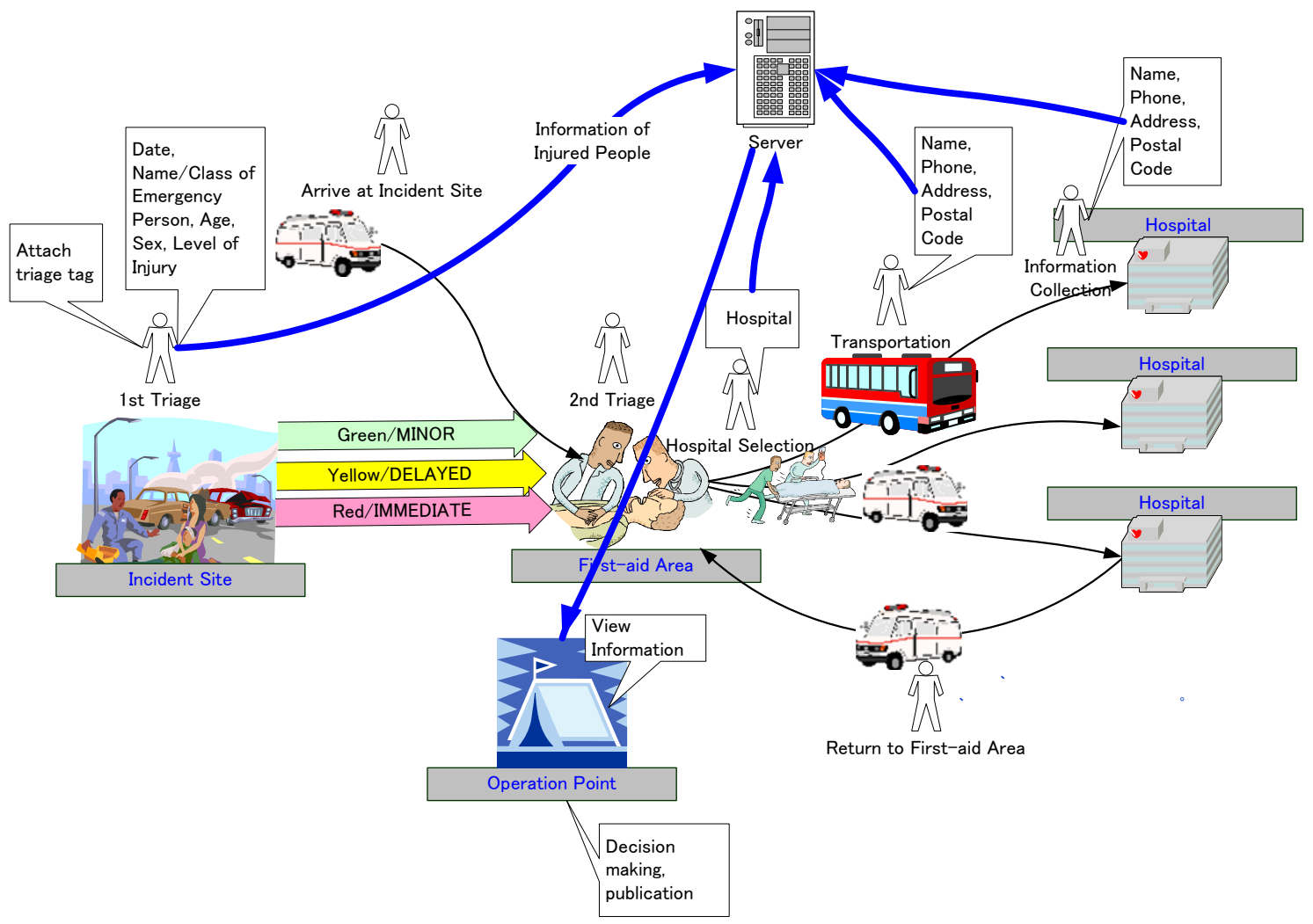

Fig. 4 shows an abstract workflow in the RFID triage system. 


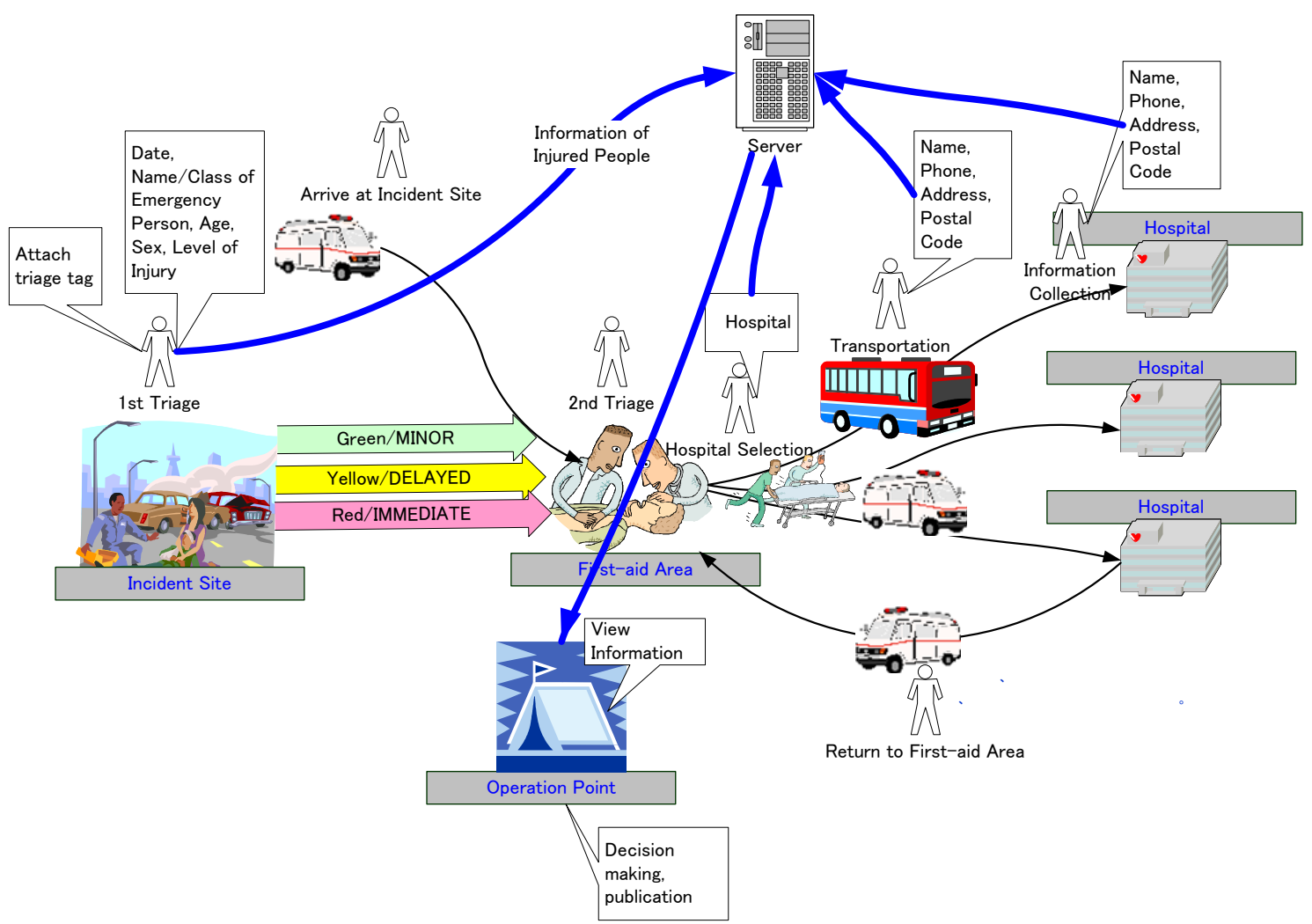

Fig. 4 Workflow in RFW Triage System.

Other than the advantages described in Section 2.3, we can employ the following advantages of the system including primitive ones:

1. Using mobile devices with wireless communication, the information of the injured people is collected quickly via the network.

2. The input method using mobile devices provides ease of reading compared to handwriting.

3. Input throughput is improved by automating the information of the emergency personnel and addresses from postal codes, and by reducing the data types necessarily required to be input in the early stages only to the injury-level and hospital.

\subsection{System Architecture}

The following are the system components, which we show the image in Fig. 5. 


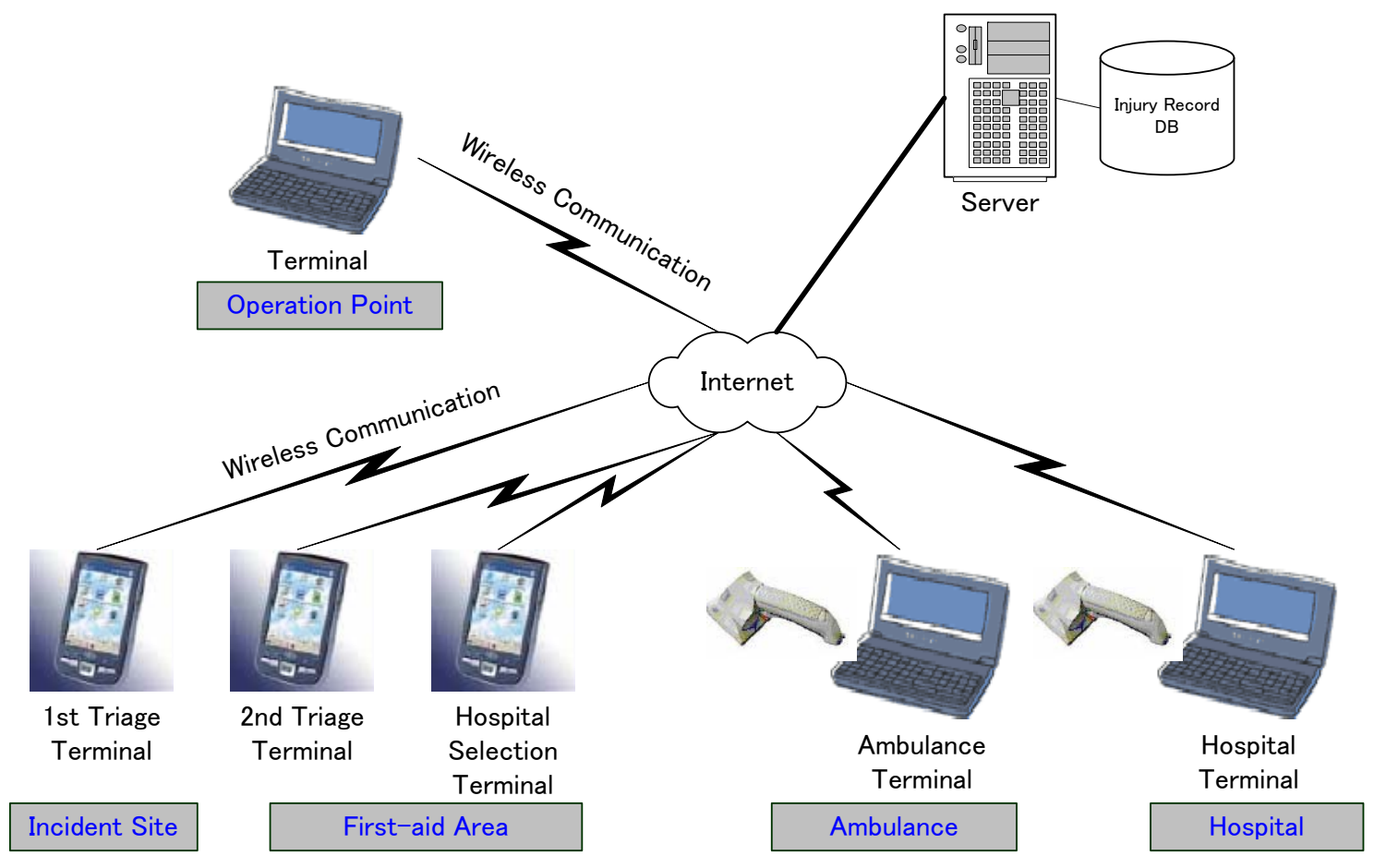

Fig. 5 The system and the network architecture

- A triage tag: a tag with input forms for the information of the injured person and an RFID tag with rewritable memory of 1 kilobyte and wireless communication in the frequency of 13.56MHz. Each RFID tag has a unique ID in the system.

- $1^{\text {st }}$ triage terminal: a mobile terminal device that an emergency person in charge of the 1 st triage stage uses. It is equipped with an RFID reader and a wireless communication interface. An emergency person can input injured peoples' information through the touch panel or buttons. After inputted, the information is saved to the RFID tag, as well as sent to the server through the wireless network. In case the network is disconnected, the information is stored on the terminal, and resent when the network is connected. Static or automatic information such as the date, the name/category of the emergency personnel, are inputted by the terminal automatically. Fig. 6 is the display of input terminal.

- $2^{\text {nd }}$ triage terminal: similar to 1 st triage terminals, a mobile terminal device an emergency person in the 2 nd triage stage uses.

- Hospital selection terminal: similar to 1st triage terminals, a mobile terminal device an emergency person in the hospital selection stage uses.

- Ambulance terminal: a notebook PC equipped with a handy RFID reader and a wireless communication interface, placed in each ambulance. Emergency personnel can input the information of injured people using a keyboard and a mouse. First several part of an address can be converted from a postal code.

- Hospital terminal: similar to ambulance terminals, a notebook PC emergency personnel in each hospital use.

- Operation point terminal : a notebook PC equipped with a wireless communication interface, placed in the operation point. Emergency personnel can browse the information collected to the server through a web browser software. 
- The server: server equipment which has a database of the injured people, placed away from the incident site. The server stores the information sent from the terminals to a database, and responses to the browsing request from the operation point terminals with HTTP protocols. Moreover, the server keeps the information of an injured person up-to-date from those sent by multiple terminals.

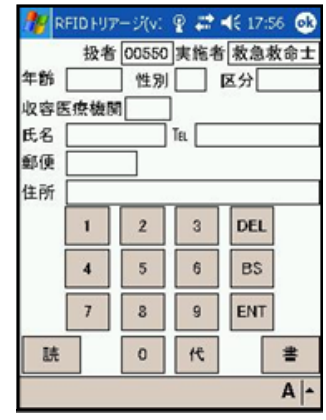

Fig. 6 Display of input terminal in $1^{\text {st }}$ and $2^{\text {nd }}$ triage stage

\subsection{The system flow}

Rough workflow of triage does not change when the system is applied. The difference is: instead of hand-writing and hand-collecting, emergency personnel read the information of each injured person from the RFID tag, input the information of each injured person to the terminal, and then writes it to the RFID tag.

In the following, we describe the usage and the behavior of the system for each role of the emergency personnel.

An emergency person in charge of $1^{\text {st }}$ triage inputs the information of each injured person as much as possible to the $1^{\text {st }}$ triage terminal, and then writes it to the RFID tag. Simultaneously, she/he attaches the triage tag with the RFID tag to the injured person, and cut off the perforated label to the corresponding color. The information of the injured person written to the RFID tag is sent to the server as soon as the network becomes available.

An emergency person in charge of $2^{\text {nd }}$ triage firstly read the information of each injured person from the RFID tag through $2^{\text {nd }}$ triage terminal, and adds/updates the information to the RFID tag by interviewing the injured person.

An emergency person in charge of hospital selection selects the hospital each injured person is transferred to, and inputs it to the RFID tag through the hospital selection terminal.

An emergency person in an ambulance or in a hospital does the same operation as $2^{\text {nd }}$ triage but using a notebook PC and a handy RFID reader to the injured person. Address is automatically complemented when a postal code is input. Additionally, the emergency person clicks the commit button on the display and records the time of carrying the injured person to the ambulance.

An emergency person in the operation point views the information of the injured people, which is collected to the server, on the operation point terminal using Web browser software, and informs them to the emergency control center in the municipality as she/he needs. The server stores the information sent from the terminals, and serves them to the operation point terminal through HTTP protocol. Multiple upload from terminals for the same injured person are sorted in time order and kept up-to-date. 
This workflow is a natural extension from current triage except the time for inputs, which we evaluated to be trivial in Section 6.5.

Tab. 1 shows the types of collected information in each stage in the current workflow or in using the system. From the table, we can observe that the information used for the publication and which needs time to input, such as name, phone number, and address, is input after the hospital selection finishes, and the emergency personnel in charge of the stage not later than hospital selection only have to input the information which is simple to input, such as the level of injury, age, sex, and hospital. Moreover, the name and the category of the emergency personnel, and the date can be input automatically or previously before the incident.

Tab. 1 Types and stages of collected information in current workflow or RFID triage system

\begin{tabular}{|c|c|c|c|c|c|c|c|c|c|c|}
\hline $\begin{array}{r}\text { Type of } \\
\text { information }\end{array}$ & Date & $\begin{array}{c}\text { Emergency } \\
\text { person }\end{array}$ & $\begin{array}{c}\text { Category } \\
\text { of } \\
\text { emergency } \\
\text { person }\end{array}$ & Age & Sex & $\begin{array}{c}\text { Level of } \\
\text { injury }\end{array}$ & Hospital & Name & $\begin{array}{c}\text { Phone } \\
\text { Number }\end{array}$ & $\begin{array}{c}\text { Address } \\
\text { Stage }\end{array}$ \\
\hline $1^{\text {st }}$ triage & AUTO & AUTO & AUTO & Y & Y & Y & - & - & - & - \\
\hline $2^{\text {nd }}$ triage & AUTO & AUTO & - & - & - & - & - & Y->N & Y->N & Y->N \\
\hline Hospital selection & - & - & - & - & - & - & $\mathrm{Y}$ & - & - & - \\
\hline Ambulance & - & - & - & - & - & - & $\mathrm{Y}$ & $\mathrm{Y}$ & $\mathrm{Y}$ & $\mathrm{Y}$ \\
\hline Hospital & - & - & - & - & - & - & $\mathrm{Y}$ & $\mathrm{Y}$ & $\mathrm{Y}$ & $\mathrm{Y}$ \\
\hline
\end{tabular}

Legend: $Y$ : input by emergency personnel both in current workflow and RFID triage system,

$\mathrm{Y}->\mathrm{N} \quad$ : not input in RFID triage system, while input in current workflow,

AUTO : automatically or previously input in the system, while input in current workflow

- $\quad$ : $\quad$ : not input in both cases

\section{Related Work}

Triage is captured as an application that requires immediate improvement in the field of emergency medicine [3][4][5][6][7][8] and is realized as one of the important applications in pervasive computing [17].

A variety of information systems can be considered to be useful to support triage, such wireless and mobile telemedicine systems[12]. Wireless network composition for such an application is discussed [13]. Tiny devices such as sensors to the network is tried to use for vital sensors for injured people [17]. Active RFID tags are tried to use for tracking the location of injured people [19].

On the other hands, information systems for supporting disaster management are proposed by featuring several key technologies in pervasive computing. Geographical information systems are used to locate and identify the context of people and objects[10]. Immediate deployment of wireless networks is discussed in [11]. RFID tags are tried to use for planning and guiding the evacuation[9].

Several work above exploit wireless networks, and several exploit RFID tags. However, these work along with networks does not address the requirements for data integrity, input throughput, availability, and latency as we shown explicitly. Moreover, RFID are not used for improving the requirements. Our approach is unique in the sense that we use 
RFID tags for the alternative of current triage tags, and for improving the requirements by alternating wireless network communication with the communication with RFID tags.

U.S. Navy tried to use rewritable RFID tags as triage tags as well as our approach do, but they are not trying to incorporate wireless network communication and RFID communication [20].

\section{Preliminary Experiment}

Prior to the massive experiment, we evaluated several points which enough with small number of participants. We measured input operations of 5 times in a room. Fig. 7--Fig. 9 are the results of the measurements.

Fig. 7 is the comparison of input times of each part in 1st triages with and without the system. From the figure, the time for an injured person is reduced to about 38 seconds from about 42 seconds. The detail shows that the most contribution for that is because the emergency personnel, with the system, do not have to input the level of injury, the name of the personnel, and the date. On the other hand, the times for preparing and cutting triage tags are increased by having input terminals in the case, and about 2.5 seconds for writing to triage tags arise.

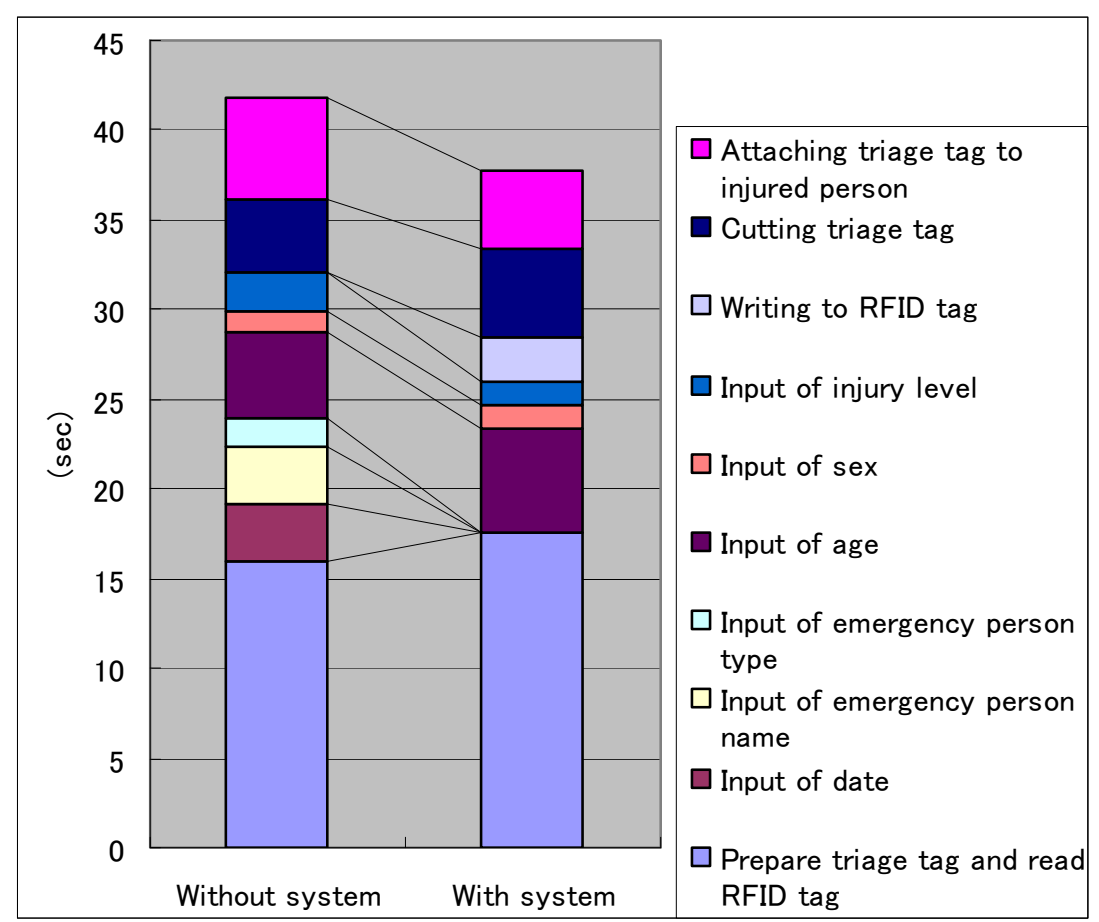

Fig. 7 Input time in $1^{\text {st }}$ triage

Fig. 8 is the comparison of input times of each part in ambulances or at hospitals with and without the system. From the figure, the time for an injured person increased to about 43 seconds from about 35 seconds. The detail shows that the address, the postal code, the phone, and the name of the injured person take longer time with the system than without the system. Although the system employs automatic address complements from postal codes, it is still longer than without 
the system. Additionally, it needs about 3.5 seconds for writing to triage tags. It is a future work to reduce the input time for ambulances and hospitals, while there exists a problem that, without the system, manual and hastened writing is often difficult to read.

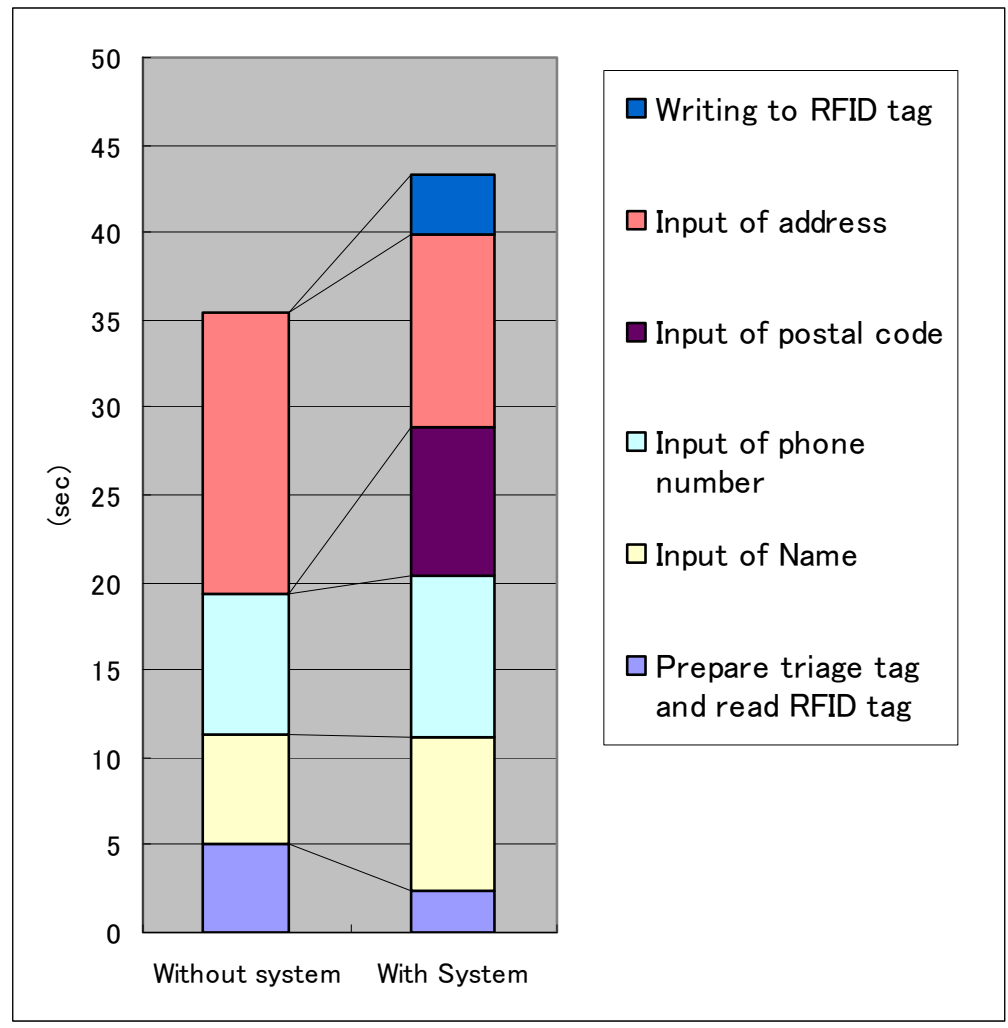

Fig. 8 Input time in ambulance and hospital

Fig. 9 is the comparison of input times of each part in hospital selections with and without the system. From the figure, the time for an injured person was reduced to about 8 seconds from about 12 seconds. The detail shows that the operations without the system require the emergency personnel to pass the copy of the triage tags to other personnel nearby, while do not with the system. On the other hand, the operations without the system can start the hospital information immediately, while in the case with the system, emergency personnel need first to read the information inputted so far from the RFID tags before input. 


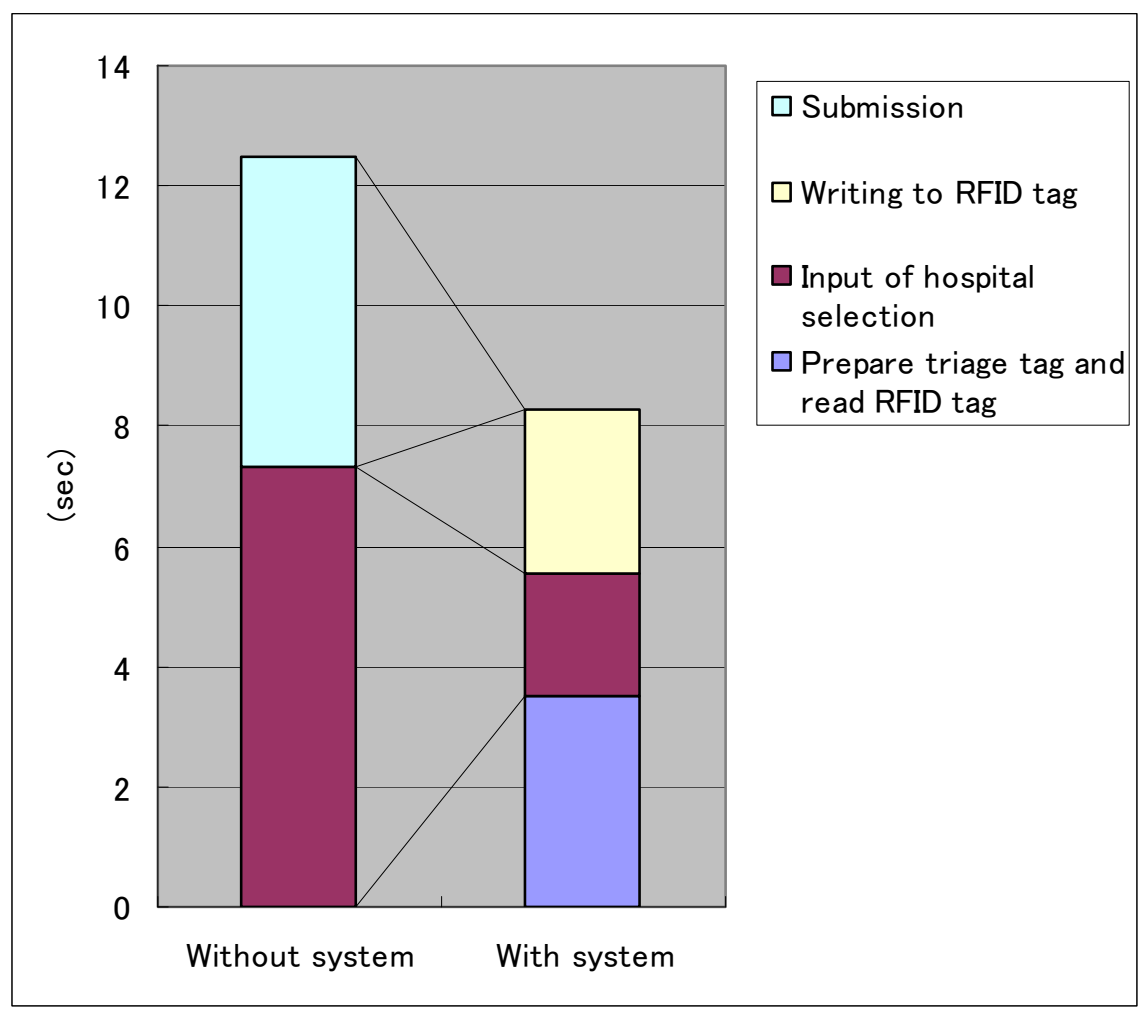

Fig. 9 Input time in hospital selection

\section{Experiment}

In this section, we describe the experiment of using the RFID triage system. The experiment is done in a driving school in a day, assuming a complex car crash of 5 cars and about 82 injured people. From the hearing from professionals of emergency operations, this population is considered to be about maximum population where current triage is feasible. The goal of the experiment is to evaluate whether and how much the RFID triage system accelerates and completes a) the transportation of the injured people and b) the collection of the injured-people's information.

\subsection{Assumption}

The experiment spot is a driving school of about 2250 square meters. In the experiment spot, we set hospitals area, which corresponds to 3 points of hospitals, and set 2 hospitals about 700 meters away from the spot.

We assume that 2 buses and 3 cars crash, where the injured people are located and classified as: 5 people as Black/DECEASED, 18 as Red/IMMEDIATE, 31 as Yellow/DELAYED, and 28 as Green/MINOR.

In the experiment, 14 ambulances ( 3 of them are for mass transportation) and 16 other vehicles such as commander cars and machinery and materials cars. The population of emergency personnel is 85 , where 4 ( 2 pairs) for $1^{\text {st }}$ triage, 1 for $2^{\text {nd }}$ triage, 1 for hospital selection, 3 for each ambulance, and 1 for each hospital. 


\subsection{System assignment}

In the experiment, two 1st triage terminal, one 2nd triage terminal, and one hospital selection terminal is used as mobile devices.

14 ambulance terminals are used, where 6 of them are equipped with RFID readers and wireless communication interfaces, 3 are equipped with RFID readers but wireless communication interface, and 5 are not with both. The ambulance terminals with no RFID readers and wireless communication interfaces are introduced to assume the heterogeneous environment here not all the ambulances supports the RFID triage system but only a usual notebook PC, such that a neighbor municipality helps the triage. The ambulances with no wireless communication interfaces are introduced to assume the situation where communication is not available.

One hospital terminal is placed at each hospital. One operation point terminal is placed at the operation point when the point is made during the experimental performance. The server is set in a data center about 1000 kilometers away from the incident site.

\subsection{Network}

We prepared IEEE 802.11b wireless LAN interfaces for mobile device terminals, and FOMA data communication interface which provides up to 385 kilo-bps by NTT Docomo Inc.

Although access point facilities is necessary if we adopt wireless LAN since otherwise it provides only several ten meters, we had to adopt it since there have been no mobile devices which is able to be equipped with an RFID reader and any long-range data communication interface of several kilometers. In the future, this cost of access point will vanish when a mobile device with an RFID reader and a long-range data communication interface.

\subsection{Process of experiment}

In the experiment, we firstly take about one hour to perform the naïve performance, which is the current triage, and then perform the advanced performance, which is the triage using the system, in the same condition.

Each performance starts with the departure of ambulances and fire engines having predefined time differences from the car parking. Each ambulance which transports the injured people to the hospitals inside the experiment spot arrives at the hospitals after driving around inside the spot, and those which transports to the hospitals outside the spot takes the predefined route.

The injured people are predefined and informed their level of injury, and replies to the questions from the emergency personnel acting according the defined level. The emergency personnel perform the triage as described in Section 2.1 or Section 3.2.

In the two performances, 7 persons measured the time of the flow of injured people, and the flow of the collected information by stopwatches. Moreover, the collected information flow was also logged in the system in the advanced performance.

\subsection{Evaluation}

The result of the experiment is shown in Fig. 11- Fig. 15. Fig. 10 is the photos of the experiment. 

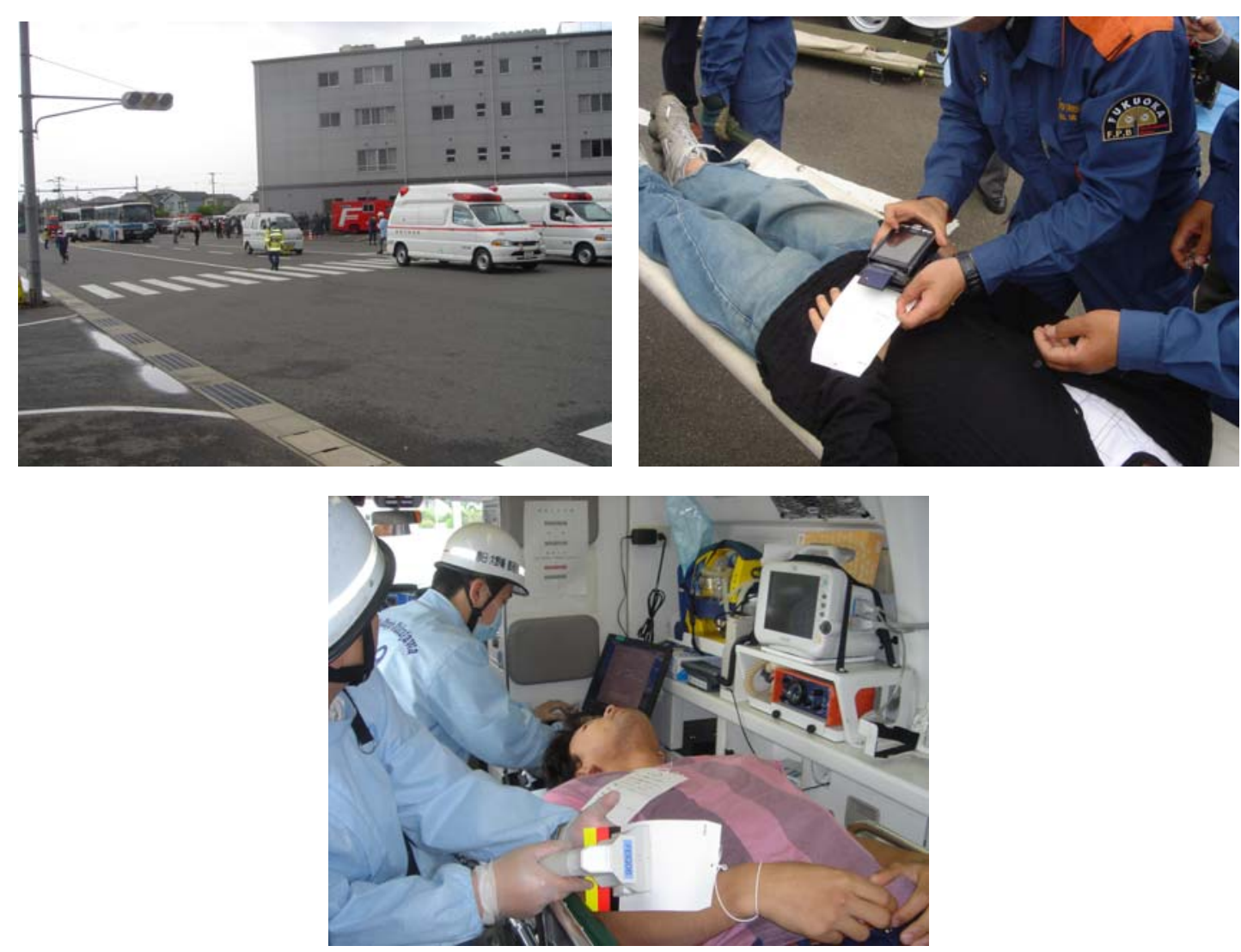

Fig. 10 Photos of the experiment

\section{Transportation of the injured people}

shows the progressions of cumulative populations of the injured people who are carried from the incident cite into the first-aid area and carried out from the first-aid area to the hospitals in each performance. 


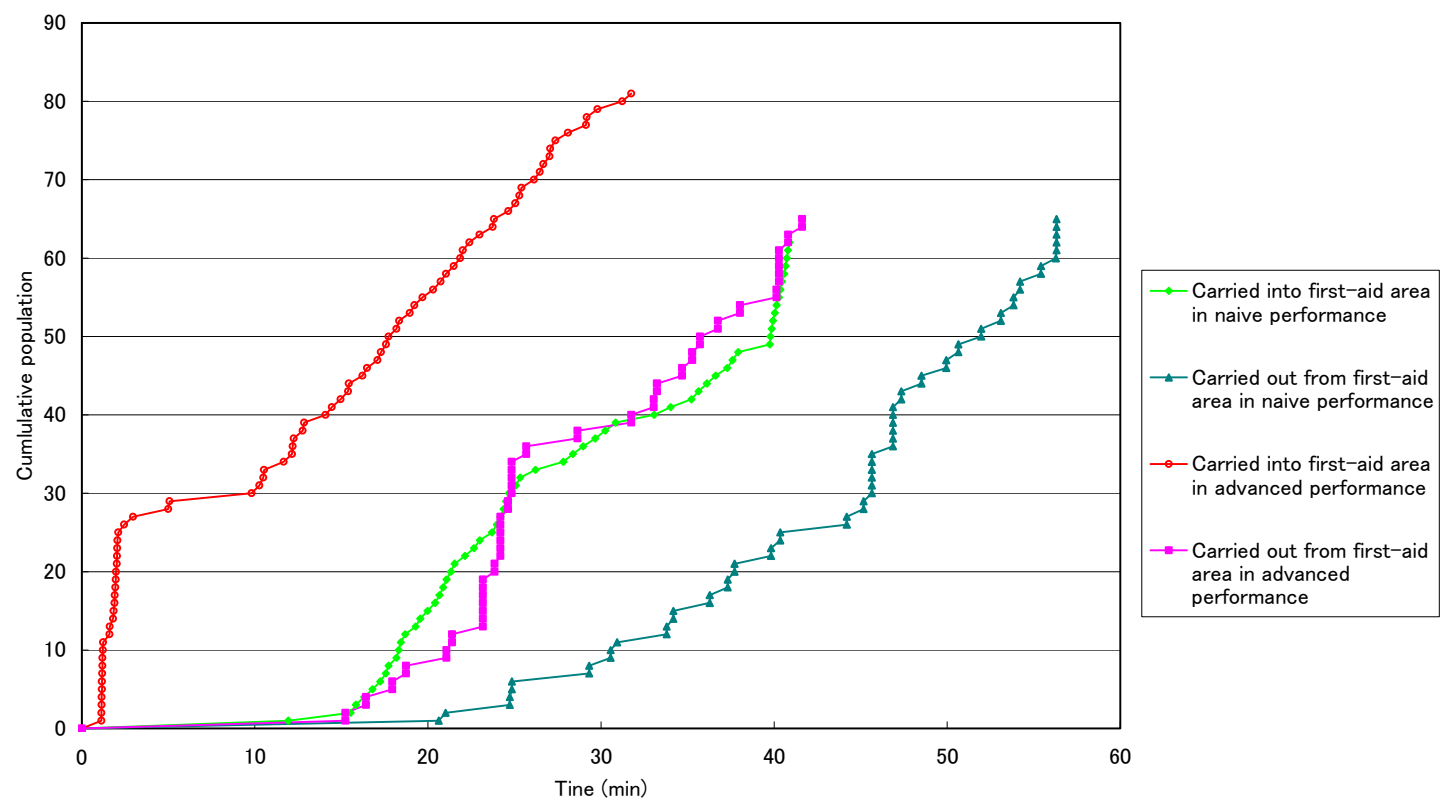

Fig. 11 Transportation Time

As to the population carried into the first-aid area in

Fig. 11, that of the advanced performance always exceeds that of the naïve performance. This demonstrates that, using the system, the injured people can be carried into the first-aid area faster than the naïve performance. This is mainly resulted by the fact that, multiple injured people could be carried in simultaneously using the system, while they must have been serial to manually count the people by emergency personnel in order not to lose the information of the injured people in the naïve performance. Moreover, the population radically increases at around 2 minutes after the start. This is the result of the parallelization of the carrying in including those who are in Green/MINOR level of injury and can walk had become possible to be carried as early as possible, while they must wait for the people of other levels have been carried in the naïve performance.

Moreover as to the population carried out from the first-aid area to the hospitals in

Fig. 11, that of the advanced performance always exceeds that of the naïve performance. This shows that, using the system, the injured people can be carried to the hospital faster than the naïve performance. This is assumed to be because, in addition to the acceleration of carrying into the first-aid area, the hospital selection was effectively performed using a mobile device in the advanced performance. Moreover, the figure shows that a plenty of injured people are carried out after 22 minutes. This is assumed to have resulted from that the injured people of Green/MINOR level could be carried out early followed by the acceleration of being carried in.

The population in naïve performance expires before it becomes the total population assumed because of the essential lack of the current workflow: several injured people in Green/MINOR level dismissed before going to the first-aid area and could not be monitored; the Black/DECEASED people was not carried into the could not be monitored, while the 
advanced performance could. Moreover, the population carried out from the first-aid-area in the advanced performance exceeds that carried into there. This is because the vehicles for Green/MINOR level could stop at a different area from the ambulances for other levels, and several people off Green/MINOR level happened to be carried out without carried into the first-aid area.

\section{Information Collection}

Fig. 12 is the progression of the cumulative populations whose information about the injury level is collected to the manual copy at the operation point in naïve performance, to the RFID tag of the injured person in the advanced performance, and the server in the advanced performance. Fig. 13 is that about the hospital selection, and Fig. 14 is that about the address. If there are multiple input records for a single type of information, we adopted the first period of the collection. The time in the server was measured by a unit of a minute while the others were by seconds.

In the following, we describe the method for recording and adjusting the record in the naïve performance. The level of injury, the sex, and the age was assumed to be collected when the entry in the triage tag is copied to a tally sheet before an injured person is transported from the first-aid area toward a hospital. The name, the address, and the phone was assumed to be so when it is copied after an ambulance returned to the operation point after the transportation. The records have been made slight complements to the deficit in the data obtained from the stopwatch measurement in the following way:

- We adopted the time of particular people for 7 lacks of time where the subject injured people could be assumed to be in the same ambulances.

- We adopted the time of the closest line in the tally sheet for 6 lacks of time where Green/MINOR people could be assumed to be transported at a time.

- For 2 time lacks of the manual copy to the tally sheet after the transportation while the times of collection are known, we adopted the collected times added by the averages of the periods from the collection to the manual copy of other people of the same hospitals.

For 1 person whose collection time after the transportation and also that of manual copy lack, we adopted the average time of the times of manual copy for the same hospital.

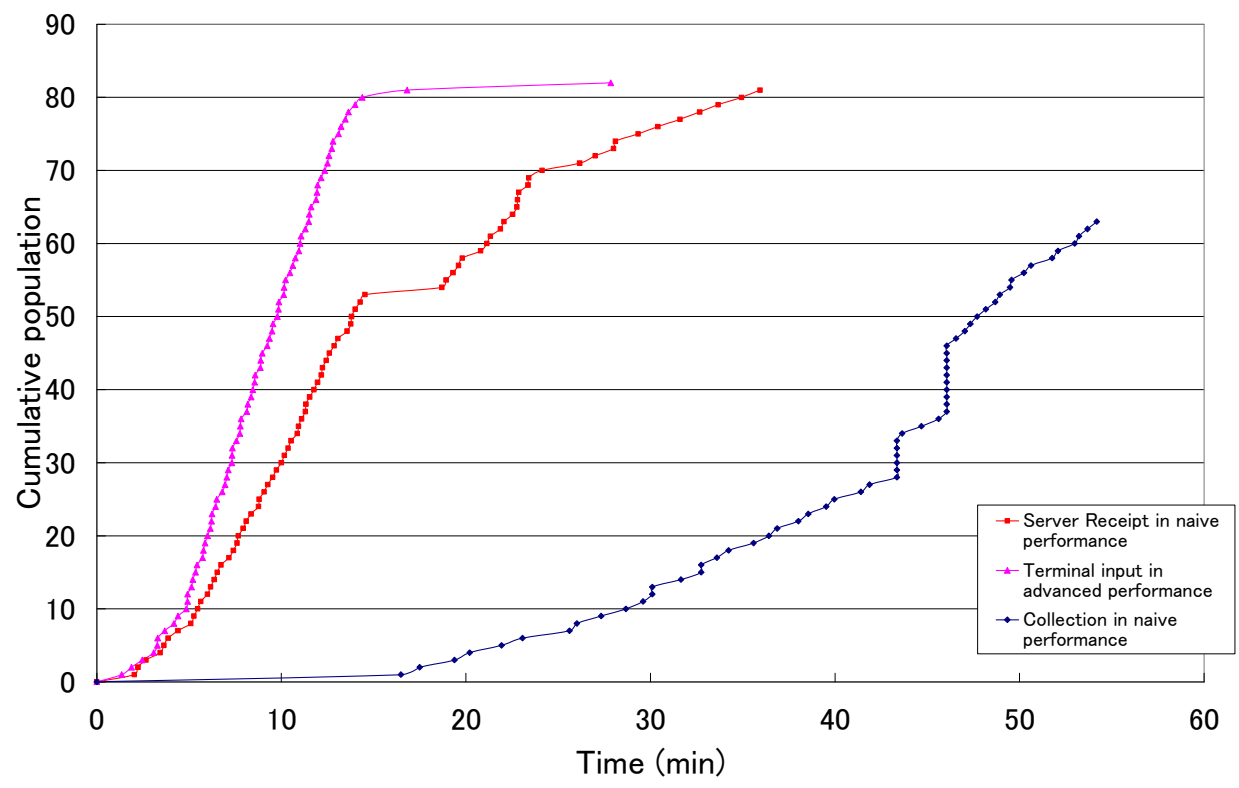


Fig. 12 Time of information collection (level of injury)

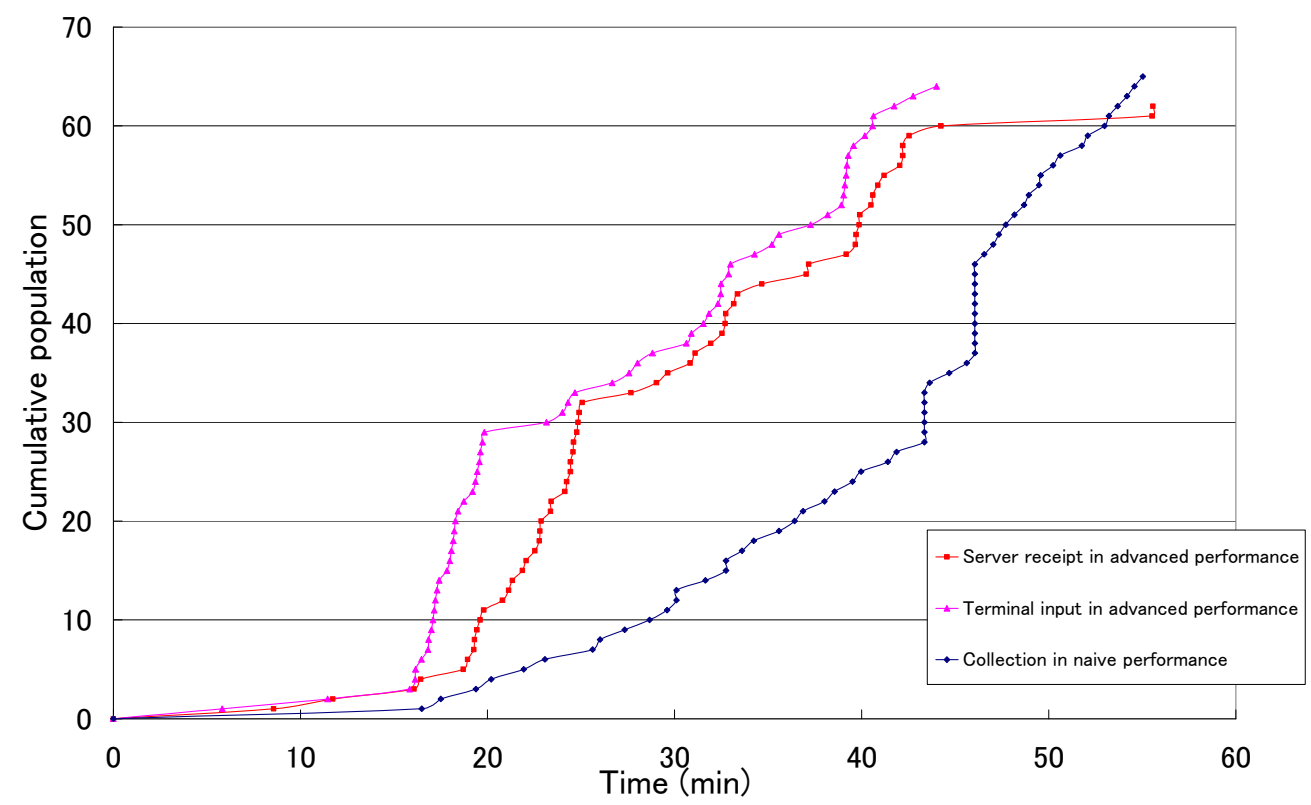

Fig. 13 Time of information collection (hospital selection)

It is not easy to compare the times of the naïve and advanced performance since the methods of measurement are different, but we can see the times for the operation point to view the information for the first time, if we consider it they correspond to the manual copies at the operation point in the naïve case, and so to the data arrivals to the server in the advanced case. 


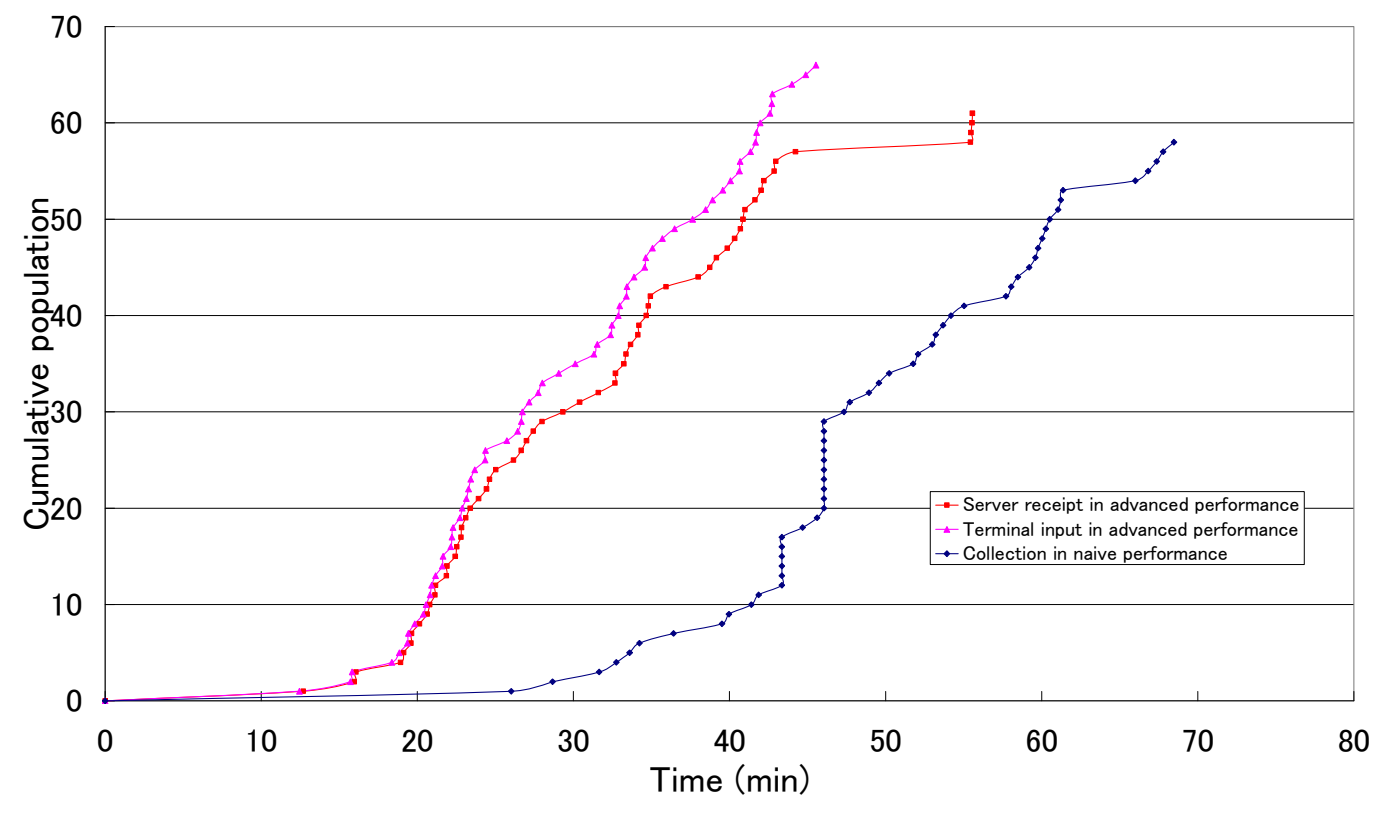

Fig. 14 Time of information collection (address)

From the figures, the information of the injured people using the system could be collected faster than the naïve performance in any information. Although we omit the graphs for the other information, there was little difference in the shapes in the graphs of level of injury, sex, and age. Similarly, so was in name, phone number, and address. These mainly owe to the timing of input shown in Tab. 1, and we can observe that the collection of the information in the early stages of the triage could radically be accelerated using the system.

Collecting information of all the injured people is essentially difficult, since that of the Black/DECEASED people are not collected quickly, and the Green/MINOR people tends to walk out on their own. However, Fig. 12 and Fig. 14 show that the number using the system reaches higher than the naïve performance, which means that the system provides more accurate and complete information of injured people especially in the early stages of triage. Although Fig. 13 does not shows the same, this can be assumed since the input time using the system was longer than the naïve performance, which we confirmed in Section 5 in which the average input time of the hospital selection in the system was about 8.2 seconds, while 7.3 seconds without the system, with 5 trials in each case. Improving the time and complexity of input, there is a possibility of making the information collection of hospital selection accurate.

For data integrity in the advanced performance, all the peoples' data have not been collected in Fig. 14 since the Black/DESEASED and Green/MINOR peoples' information have not been collected. However, the number of collection is more than the naïve case. In Fig. 13, the number of collection in the naïve case is better than the advanced case. This can be assumed because the trial with the system needs, except for submission, much time than without the system as shown in Section 5.

\section{Communication time in the system}

From Fig. 12-Fig. 14, we can observe that the network communication time in the system does not affect the triage to the critical delay. Besides, the receipt time on the server can be earlier if the times for communication are reduced, although 
the result in the advanced performance is better than the naïve one. .Fig. 15 is the distribution of the difference between the time received by the server and that input to the input terminal of each input of the injury information, where the differences of more than 60 seconds are omitted as a device without wireless communication functionality. The average time of the difference between the time received by the server and that input to the terminal was 17.3 seconds, and the variance was 35.39 .

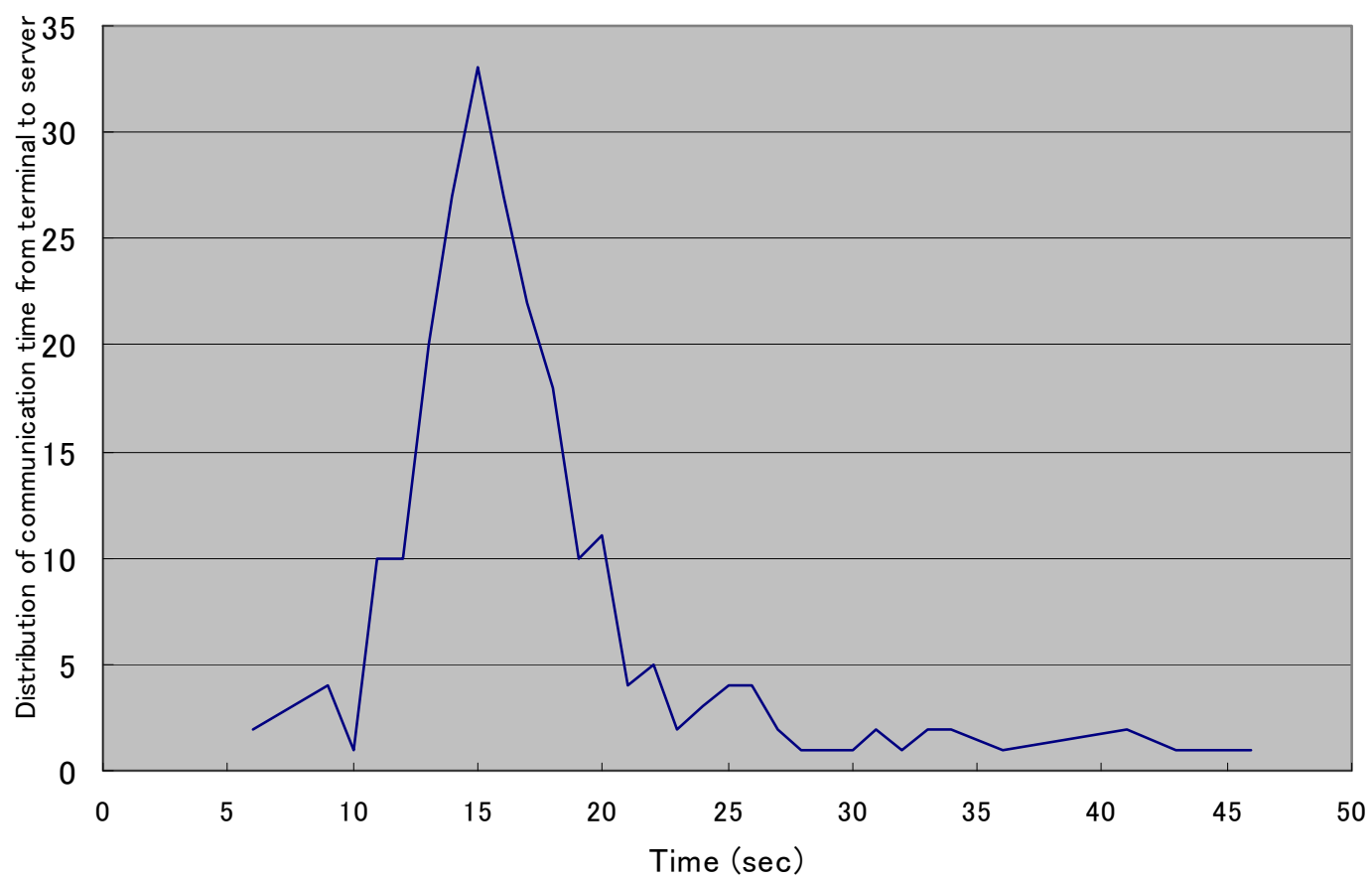

Fig. 15 Distribution of communication time in the system

\subsection{Discussion}

We discuss the implications in network with the requirements discussed in Section 2.

Data integrity and availability are apparent to be improved without the experiment, as discussed in Section 2.3.

As to input throughput, note that the communication time does not affect the input time by the emergency personnel, since they firstly read the old information from an RFID tag, and finishes right after updating the information. The terminal automatically communicates with the server subsequently, but the emergency personnel do not have to wait the network communication. If the system does not employ RFID tags, the first step of reading from the RFID tag must rely to the network communication. The throughput of an input by discarding RFID tags can be supposed to increase by 14.3 seconds, since the network communication time is 17.3 seconds in average and the user-waiting time for the RFID communication is 3 seconds as shown above.

Although latency needs further research to clarify the advantage of using RFID tags, we could decrease the latency, especially the required types of information compared to the naïve performance as shown in Figure 3 and 4, without affecting other factors such as input throughput in triage. 


\section{Conclusion}

From the experiment, the RFID triage system is demonstrated to be effective in mass casualty incidents of about 100 injured people. In the future, the system will be more valuable when it becomes applicable for other incidents, such as with smaller number of injured people, or in broad area, such as earthquakes, storms, or floods.

\section{Acknowledgement}

This work has been supported by the Grant-in-Aid for Young Scientists (A) No.18680009 of the Ministry of Education, Science, Sports and Culture (MEXT) from 2006 to 2008. We are grateful for the support of the experiment from Toppan Printing Co., Ltd., Fukuoka city, Mizuho Information \& Research Institute Inc., Institute of Systems \& Information Technologies/KYUSHU, NTT DoCoMo, Inc., NTT West Corp., and Kasuga-Ohnojo-Nakagawa Fire department.

\section{References}

[1] P. Hewkin, "Smart Tags - The Distributed Memory Revolution," IEEE Review, 1989.

[2] R. Want, K. P. Fishkin, A. Gujar, B. L. Harrison, "Bridging Physical and Virtual Worlds with Electronic Tags," Proc. Int'l Conf. CHI 99, pp. 370-377, 1999.

[3] F. Subash, F. Dunn, B. McNicholl, J. Marlow, “Team triage improves emergency department efficiency," Emerg. Med. J.21, pp542-544, 2004 Sep.

[4] T. Kilner, "Triage decisions of prehospital emergency health care providers, using a multiple casualty scenario paper exercise,” Emerg. Med. J., 19, pp.348 - 353, Jul 2002.

[5] S. Goodacre, F. Morris, B. Tesfayohannes, G. Sutton, "Should ambulant patients be directed to reception or triage first?,” Emerg. Med. J., 18, pp.441 - 443, Nov 2001.

[6] J Terris, P Leman, N O’Connor, R Wood, “Making an IMPACT on emergency department flow: improving patient processing assisted by consultant at triage,” Emerg. Med. J., 21, pp.537 - 541, Sep 2004.

[7] White, Ann, “Change strategies make for smooth transitions,” Nursing Management. 35(2) pp.49-52, Feb. 2004

[8] Parker, Pam, "Move care to a higher level with emergency systems,” Nursing Management. 35(9), pp.82-84, Sep. 2004.

[9] S. Sharma, S. Gifford, "Using RFID to Evaluate Evacuation Behavior Models," North American Fuzzy Information Processing Society Conference, 5 pages, 2005.

[10] P. Oosterom, S. Zlatanova, S. Fendel, M. Elfriede (Eds.) “Geo-information for disaster management,” Springer Verlag, 1434, 2005.

[11] S. Midkiff, C. Bostian, "Rapidly-Deployable Broadband Wireless Networks for Disaster and Emergency Response” 1st IEEE Workshop on Disaster Recover Networks (DIREN '02), 10 pages, Jun. 2002.

[12] S. Voskarides, C. Pattichis, R. Istepanian, E. Kyriacou, M. Pattichis, C. Schizas, "Mobile Health Systems: A Brief Overview,” Proc. SPIE AeroSense 2002, pp. 124-131, 2002.

[13] K. Banitsas, R. Istepanian, S. Tachakra, T. Owens, "Modelling issues of Wireless LANs for Accident and Emergency Departments,” IEEE EMBC Conf., vol. 4, pp. 3540-3543, Oct 2001.

[14] M. Gerla, J. Tsai, “Multicluster, mobile, multimedia radio network,” ACM/Baltzer Journal of Wireless Networks. vol. 1, (no. 3), pp. 255-265, 1995.

[15] G Zussman, A. Segall, "Energy Efficient Routing in Ad Hoc Disaster Recovery Networks,” $22^{\text {nd }}$ Conf. IEEE Computers and Communciation Societies(INFOCOM), 10 pages, 2003.

[16] T. ElBatt, S. Krishnamurthy, D. Connors, S. Dao, "Power Management for Throughput Enhancement in Wireless Ad-Hoc Networks,” Proc. IEEE ICC 2000, 9 pages, 2000. 
[17] K. Lorincz, D. Malan, T. Fulford-Jones, A. Nawoj, A. Clavel, V. Shnayder, G Mainland, M. Welsh, “Sensor Networks for Emergency Response: Challenges and Opportunities,” IEEE Pervasive, Vol. 3, Num. 4, pp.16-23, 2004.

[18] S. Inoue, D. Hagiwara, H. Yasuura, “A Systematic Approach for the Reliability of RFID Systems," Proc. IEEE TENCON 2004, pp.183-186, Nov. 2004.

[19] http://www.activewaveinc.com/applications_hospitals.html

[20] J. Yoshida, “Navy to Use RFID Technology in Iraq,” EE Times News, May, 2003, http://www.embedded.com/showArticle.jhtml?articleID=10700142.

Sozo Inoue is an associate professor in Research and Development Division, Library, Kyushu University. His research interests include RFID information systems, security, privacy and reliability in RFID systems, and database systems. Inoue has a Doctor of Engineering from Kyushu University. He is a member of the IEEE Computer Society, the ACM, the Database Society of Japan (DBSJ), and the Information Processing Society of Japan (IPSJ). Contact him at sozo@acm.org.

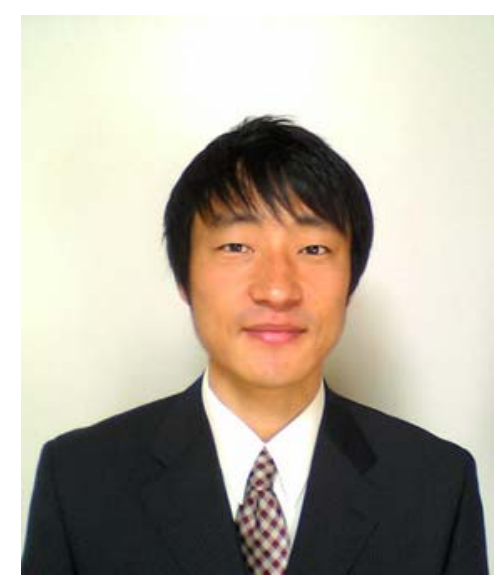

Akihito Sonoda is a student in Graduate School of Information Science and Electrical Engineering, Kyushu University. His research interests include RFID information systems, security, privacy and reliability in RFID systems, and system LSI. Sonoda has a Bachelor of Engineering from Kyushu University. Contact him at sonoda@c.csce.kyushu-u.ac.jp. 
? 